\title{
Effect of the flow velocity on bubble boiling characteristics
}

\author{
Levin Anatoliy", and Khan Polina \\ Melentiev Energy Systems Institute SB RAS, 664033, Lermontova St., 130, Irkutsk, Russian Federation
}

\begin{abstract}
The present research considers the initial stage of nucleate boiling with high heat fluxes releasing from the technical surface. We show new experimental data on the dynamics of the vapor phase in subcooled water flow in the channel under nonstationary heat release conditions. The heat release dissipation on the heater was performed by passing a controlled three-phase rectified electric current through a tube with a pulse duration of $\tau_{i \mathrm{mp}}=60-300 \mathrm{~ms}$ with a heating rate of $1000-6000 \mathrm{~K} / \mathrm{s}$. We studied the formation dynamics and the structure of the vapor-liquid layer near the heat releasing wall and monitored the temperature state of the wall depending on the parameters of the heater's flow and the intensity of heating of the cylindrical wall.
\end{abstract}

\section{Nomenclature}

$N_{a}-$ nucleation density, $1 / \mathrm{mm} 2$

$p_{0} \quad$ - initial pressure, MPa;

$T_{\mathrm{w}}-$ surface temperature, $\mathrm{K}$;

$T_{\mathrm{s}} \quad-$ saturation temperature, $\mathrm{K}$;

$w$ - flow velocity, $\mathrm{m} / \mathrm{s}$

$\Theta_{l}-$ dimensionless liquid temperature, $T_{l} / T_{0}$;

$T_{l} \quad$ - liquid temperature;

$T_{0} \quad$ - inlet liquid temperature;

$\Delta T_{\text {sub- }}$ subcooling level, $T_{\mathrm{s}}-T_{l}, \mathrm{~K}$;

$\tau_{\text {ind }}-$ induction time for intensive evaporation, s;

$\tau_{\text {imp }}$ - duration of the heat release, s;

$\tau_{\mathrm{b}} \quad-$ duration of the single nucleation, $\mathrm{s}$.

\section{Introduction}

Studies of liquid boiling processes under the conditions of the beginning crisis remain relevant due to the present lack of convincing methods for calculating unsteady boiling [1]. Thus, at the stage of bubble boiling, it is impossible to quantitatively determine the evaporation centers density [2], which is a key value for calculating the heat transfer coefficient and the onset time of the intense vapor formation. In this regard, we face the problem of expanding the experimental data on the conditions of onset and development of heat-exchange crisis modes. Currently, there is a number of papers addressing the experimental study of unsteady boiling on thin-wire heaters $[3,4]$. However, this study is focused on the development stages a heat exchange crisis under unsteady conditions on large-sized heaters.

\section{Experimental setup}

Experiments on adding power to a heat releasing channel with forced flow were carried out on a specially manufactured flow channel with a central heating pipe of 321 stainless steel (diameter $12 \mathrm{~mm}$, surface roughness $4 \mu \mathrm{m}$ ), optically transparent windows and equipped with sensors to measure temperature, pressure and flow rate integrated into the High Temperature Circuit. The scheme of the experimental setup was described in the $[5,6]$. The heat release on the heater was carried out by passing a controlled three-phase rectified electric current through a tube with a pulse duration of $\tau_{\mathrm{imp}} 60-300 \mathrm{~ms}$ and with a heating rate of $1000-6000 \mathrm{~K} / \mathrm{s}$. The initial pressure $p_{0}$ in the channel was $0.1-0.7 \mathrm{MPa}$. The magnitude of the liquid subcooling to saturation temperature was varied from $60 \mathrm{~K}$ to $135 \mathrm{~K}$. We studied the dynamics of formation and the structure of the vaporliquid layer near the heat releasing wall, temperature state of the wall (depending on the parameters of the coolant flow) and the heating intensity of the cylindrical wall. The process was visualized at a shooting frequency from $2000 \mathrm{~Hz}$ to $30000 \mathrm{~Hz}$. Video frames of boiling water and ethanol were analyzed, as well as signals from pressure sensors detecting dynamics during intensive expansion of the vapor phase.

Unsteady boiling of subcooled water is a two-stage process. The growth of bubbles with a detachment from the wall is preceded by the "pseudo-boiling" stage, when microbubbles with a diameter up to $0.1 \mathrm{~mm}$ appear and collapse with great frequency on a heat-releasing wall cooled with a liquid heated to the saturation temperature.

Due to this, heat transfer to water increases. The transition to a developed boiling on the heat releasing surface occurs after the required temperature of subcooling of the liquid layer adjacent to the surface is reached and the formation of relatively large vapor nuclei of about $1 \mathrm{~mm}$ in size begins. The time interval between the beginning of the power release and the start of intensive vaporization on the heat releasing wall is characterized by an induction period $\tau_{\text {ind. }}$. The second stage, $\tau>\tau_{\text {ind }}$, is characterized by a rapid growth and 


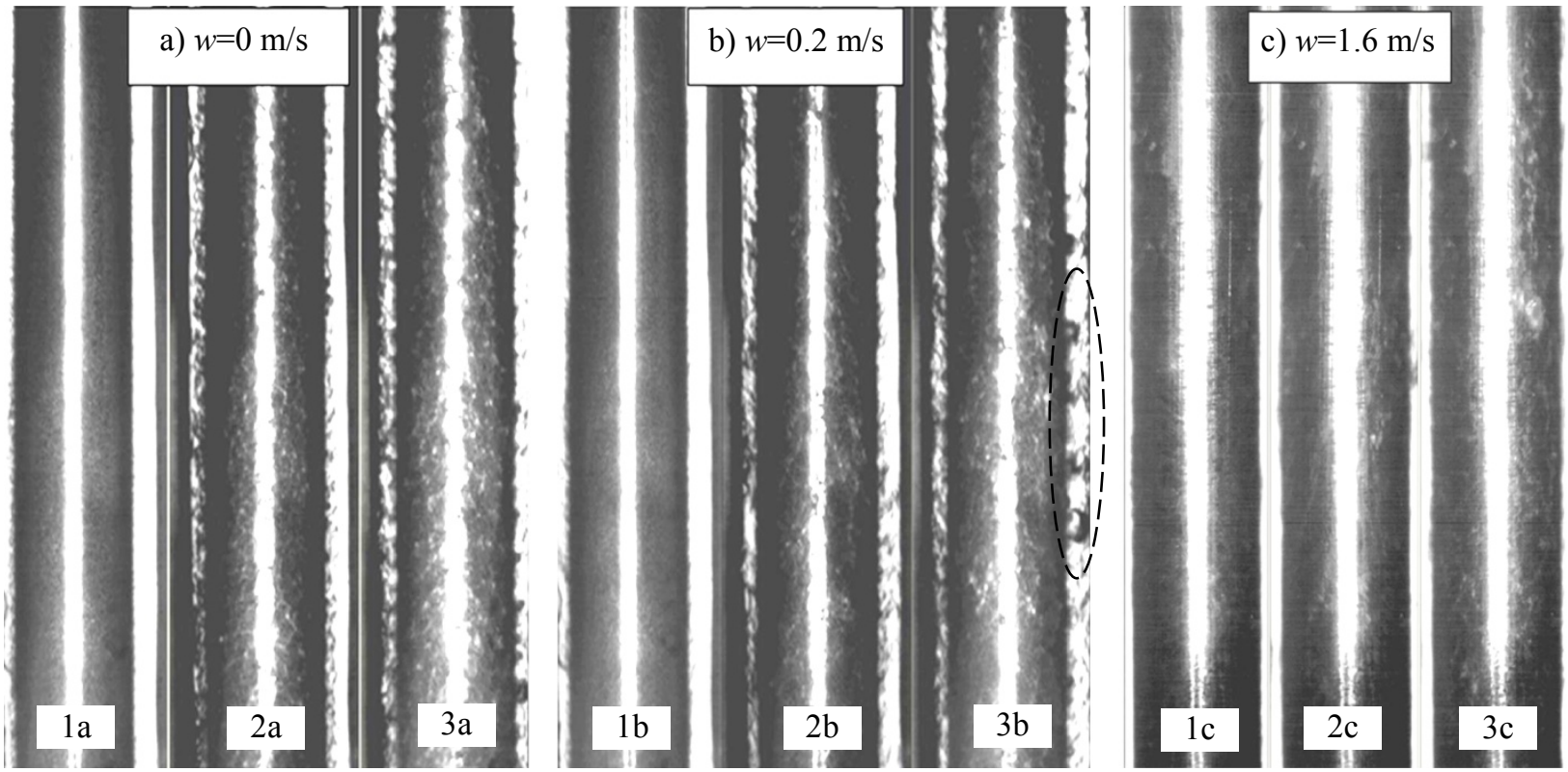

Fig. 1. Water boiling dynamics at the heater's surface for different flow velocities. 1 - before heating. $\tau=0 \mathrm{~ms}, 2-\tau=60 \mathrm{~ms} ; 3-$ $\tau=100 \mathrm{~ms}$.

agglomeration of growing bubbles into vapor conglomerates, which are subsequently carried away by a liquid flow, and is accompanied by an increase in pressure. This allows us to identify the beginning of the pressure growth in the liquid, recorded by highfrequency pressure sensors, with the onset of unsteady water boiling on the heat releasing wall.

The experiments showed a non-monotonic dependence of the induction time on the beginning of the intensive growth of bubbles $\tau_{\text {ind }}$ on the flow rate of the water under the boiling point of the water under conditions of increasing wall temperature. The experiments showed that intensive evaporation induction time $\tau_{\text {ind }}$ non-monotonously depend on the subcooled water flow velocity when the wall temperature grows rapidly. On the video frames shown in Fig. 1, we can see that vapor bubbles in a liquid moving at velocity

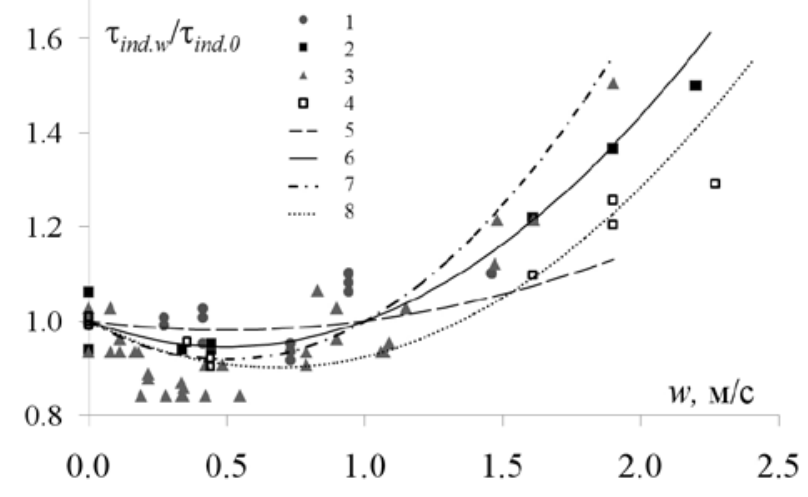

Fig. 2. Effect of flow velocity on the induction time for intensive evaporation. Experiment conditions: $1-\Delta T_{\mathrm{sub}}=65$ $\mathrm{K}, d T_{w} / d \tau=2300 \mathrm{~K} / \mathrm{s} ; 2-\Delta T_{\mathrm{sub}}=110 \mathrm{~K}, d T_{w} / d \tau=2300 \mathrm{~K} / \mathrm{s} ; 3-$ $\Delta T_{\text {sub }}=135 \mathrm{~K}, d T_{w} / d \tau=2300 \mathrm{~K} / \mathrm{s} ; 4-\Delta T_{\mathrm{sub}}=110 \mathrm{~K}$,

$d T_{w} / d \tau=3100 \mathrm{~K} / \mathrm{s}$. Calculation by (1): $5-\Delta T_{\mathrm{sub}}=65 \mathrm{~K}$, $d T_{w} / d \tau=2300 \mathrm{~K} / \mathrm{s} ; 6-\Delta T_{\mathrm{sub}}=110 \mathrm{~K}, d T_{w} / d \tau=2300 \mathrm{~K} / \mathrm{s} ; 7-$ $\Delta T_{\mathrm{sub}}=135 \mathrm{~K}, d T_{w} / d \tau=2300 \mathrm{~K} / \mathrm{s} ; 8-\Delta T_{\mathrm{sub}}=110 \mathrm{~K}$, $d T_{w} / d \tau=3100 \mathrm{~K} / \mathrm{s}$ $w=0.2 \mathrm{~m} / \mathrm{s}$ (Frame 3b) start to form earlier than in the experiment with zero water velocity (Frame 3a). As the velocity increases to $w=1.6 \mathrm{~m} / \mathrm{s}$, the formation of vapor bubbles slows down (Frame 3c), and their active growth occurs later.

The experimental results were generalized by the following approximate dependency:

$$
\tau_{\text {ind }} / \tau_{\text {ind } .0}=1+1.8 \cdot 10^{-5} \Delta T_{\text {sub }}^{2}\left(w^{2}-\frac{d T_{w}}{d \tau} w\right),
$$

where $\tau_{\text {ind. } 0}$ is the induction time for the beginning of boiling under the conditions $w=0 \mathrm{~m} / \mathrm{s}$. Formula (1) was obtained for the subcooling range $\Delta T_{\text {sub }}=65-135 \mathrm{~K}$, for the heating velocity of the heat releasing surface $\mathrm{d} T_{\mathrm{w}} / \mathrm{d} \tau=1600-3100 \mathrm{~K} / \mathrm{s}$ and for the water velocity $w=0-2 \mathrm{~m} / \mathrm{s}$. Figure 3 shows the dependency of the water velocity for various $\Delta T_{\text {sub }}$ and $\mathrm{d} T_{\mathrm{w}} / \mathrm{d} \tau$ with the initial pressure in the channel $p_{0}=0.7 \mathrm{MPa}$.

Figure 3 shows the dynamics of the specific density of active vaporization centers $N_{\mathrm{a}}$ on the heater surface for various flow velocities. Within the range of the

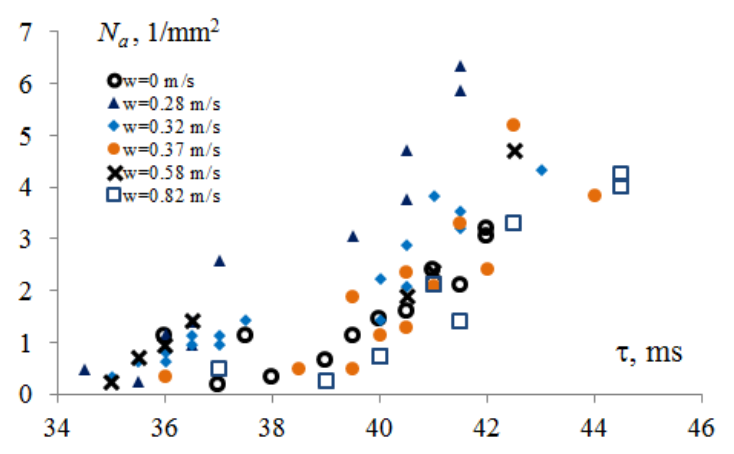

Fig. 3. Effect of flow velocity on the nucleate density. Experimental conditions: $P_{0}=0.12 \mathrm{MPa}, \mathrm{d} T_{\mathrm{w}} / \mathrm{d} \tau=$ $1650 \div 1800 \mathrm{~K} / \mathrm{s}$ 


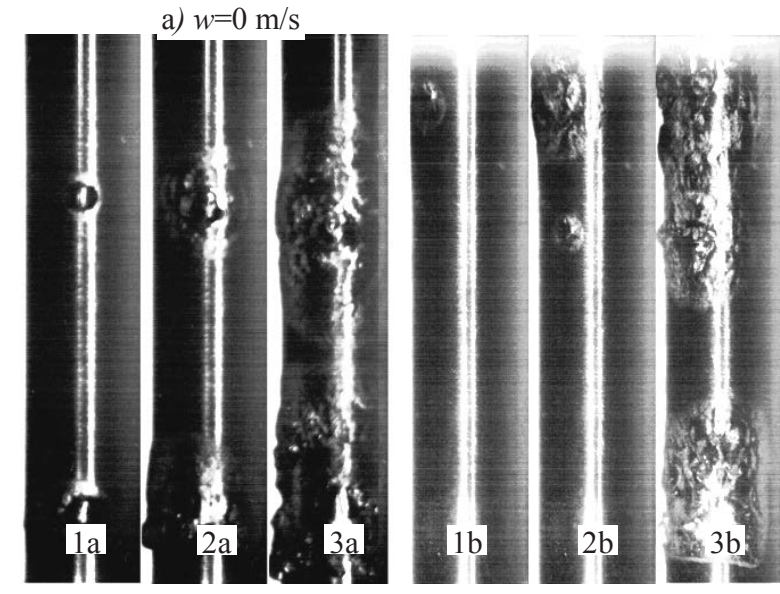

Fig. 4. Ethanol boiling dynamics at the heater's surface for different flow velocities

experimental data presented, there is no significant difference in the moment at which the first bubbles appear. However, the magnitude of the current velocity has a clear effect on the further vaporization dynamics. Thus, the boiling modes with a flow velocity $w=0.28-$ $0.37 \mathrm{~m} / \mathrm{s}$ are characterized by a higher speed at which the surface gets covered by bubbles. At the same time, a further increase in the initial flow velocity results in the opposite effect, which corresponds to an increase in the induction time $\tau_{\text {ind. }}$. Here, because of convection, the condensation intensity is enhanced due to the fact that the growing vapor bubble uplifts the surrounding thermal liquid layer.

Reduction of the induction time for the growth of bubbles under conditions of low water flow velocity (Fig. 1 and 3) can be explained by intensification of the heating of the near-wall layer in the convective "pseudoboiling" mode. This also explains the flattening of the $\tau_{\text {ind }} / \tau_{\text {ind.0 }}$ ratio curve observed in the experiments with a decrease in the water subcooling (Fig. 2).

In experiments with ethanol, the effect of the liquid velocity on $\tau_{\text {ind }}$ was not identified (Fig. 4). An increase in the initial velocity only led to the suppression of the boiling process, gradually increasing the induction time $\tau_{\text {ind. }}$ On the video frames presented in Fig. 4 , it can be seen that in the conditions of the lifting liquid flow, the onset of single bubbles formation shifts downstream. However, there is no clearly expressed visual difference in the intensity of vaporization at $w=0 \mathrm{~m} / \mathrm{s}$ and $w=0.5 \mathrm{~m} / \mathrm{s}$. The value of the initial velocity also did not have a noticeable effect on the evaporation centers density.

Such difference in the contribution of the convective component into the process of nucleate boiling of ethanol and water is stipulated by different properties of liquids that determine the mechanisms of unsteady boiling. The main difference between the mechanism of ethanol boiling is a small number of nucleation centers.

Once the required superheated temperature in the layer of ethanol adjacent to the heater is reached, the formation and growth of vapor bubbles begins. As the bubbles grow, a thin vapor film is formed in the lower part - the evaporation front, which further spreads

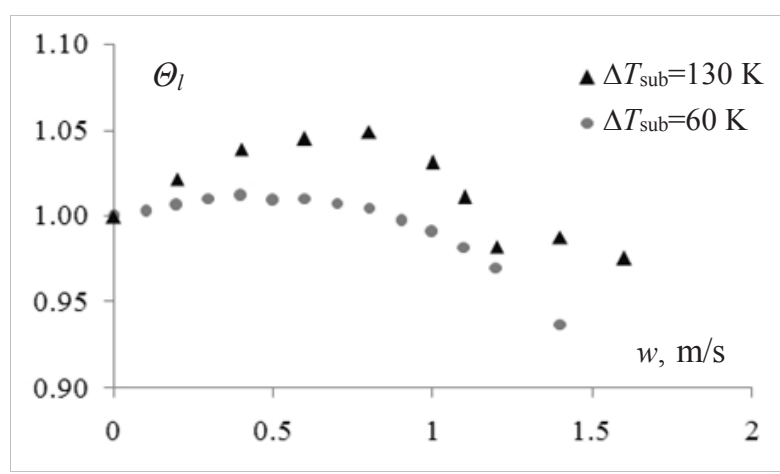

Fig. 5. Effect of the initial flow velocity on the dimensionless boundary temperature $\Theta_{l}$

around the bubble that continues to grow. Precisely from this moment an intensive increase in the vapor phase begins, leading to the crisis onset, as well as to an increase in liquid pressure. Thus, there is no "pseudoboiling" mode for ethanol; the intensification of heat exchange in which is possibly realized for certain values the forced flow velocity.

\section{Numerical modeling and analysis}

To estimate the effect of the convective component, the calculation of the unsteady liquid flows (water, ethanol) was performed under conditions similar to the experimental ones. The numerical implementation was developed in the Comsol Multiphysics package as a model of conjugate heat exchange for a cylindrical tube with a laminar liquid flow at a given value of the heat source:

$$
\begin{gathered}
\frac{\partial \rho}{\partial \tau}+\nabla \cdot(\rho w)=0, \\
\rho \frac{\partial w}{\partial \tau}+\rho w \cdot \nabla w= \\
=\nabla \cdot\left(-p+\mu\left(\nabla w+(\nabla w)^{T}\right)-\frac{2}{3} \mu(\nabla \cdot w)\right)+F_{g}, \\
\rho c_{p} \frac{\partial T}{\partial \tau}+\rho c_{p} w \nabla T=\nabla \cdot(k \nabla T)+Q
\end{gathered}
$$

where $Q$ is a heat source, $F_{g}$ is a gravity force.

Equations (2) were solved for unsteady heat generation on the wall with respect to three variables: flow velocity $w$, medium temperature $T$, and pressure $\mathrm{p}$. The calculated temperature field includes two subregions: the heater's wall with internal heat sources and the surrounding liquid. The initial velocity distribution over the channel volume was determined by a preliminary calculation of the stationary mode. The calculation was performed in an axisymmetric representation of the model, which allowed us to significantly increase the number of elements of the computational grid (1 million elements in the computational plane). Direct solver MUMPS was used to numerical solution. Calculations were performed for the following conditions: $p_{0}=0.6 \mathrm{MPa}, \Delta \mathrm{T}_{\mathrm{sub}}=60,130 \mathrm{~K}$. The heat source power value was chosen so that the heating 
rate in the free convection corresponded to $\mathrm{d} T_{\mathrm{w}} / \mathrm{d} \tau=2000 \mathrm{~K} / \mathrm{s}$.

Analysis of the calculation results of the thermal boundary layer dynamics for the water velocity range $w_{0}=0-2 \mathrm{~m} / \mathrm{s}$ confirmed the hypothesis of intensification of the near-wall layer heating due to convective transfer in the region of moderate velocities. In Figure 5, we can see a local increase in the ratio of the near-wall liquid layer temperature to the layer temperature under free convection conditions $\Theta_{1}=T_{1} / T_{0}$ at the distance of $0.05 \mathrm{~m}$ from the beginning of the heating zone.

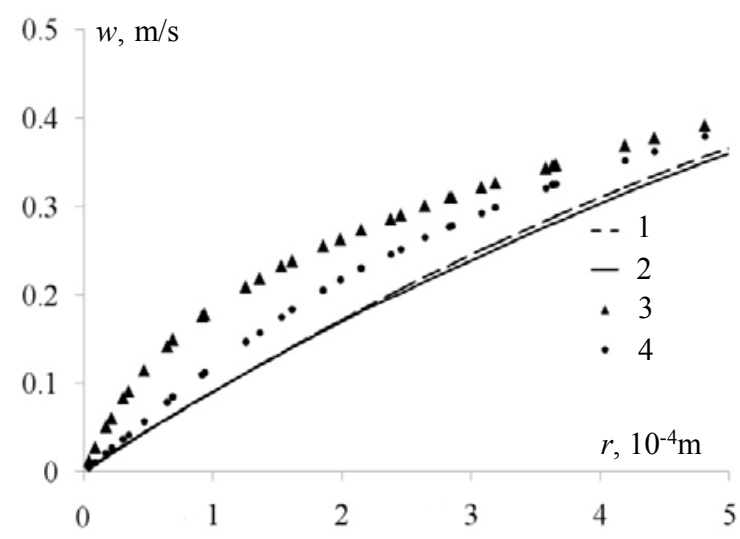

Fig. 6. The calculated flow velocity in the boundary layer. 1 - no heating, water; 2 - no heating, ethanol; 3 - water in $100 \mathrm{~ms}$ after heat release; 4 - ethanol in $100 \mathrm{~ms}$ after heat release

A faster heating of the near-wall liquid layer leads to an early achievement of the temperature required for the start of intensive vaporization, as can be seen in Fig. 1. We can also note the effect of liquid subcooled to the saturation temperature (the character of subcooling coincides with that observed in the experiments (Fig. 2)): an increase in subcooling leads to intensification of the heating of the near-wall liquid layer.

We also calculated the dynamics of profiles for the water and ethanol flow velocities, which helped to estimate the change in the hydrodynamic layer thickness during the heating of the near-wall liquid layer. Figure 6 shows the instantaneous isolines of the water and ethanol velocities in $100 \mathrm{~ms}$ from the beginning of the power release. We can observe a significant difference between the change in the flow velocity profile for water and ethanol. This explains the insignificant contribution of convection into the conditions for transition to the boiling crisis for ethanol.

\section{Conclusions}

We revealed the non-monotonous dependence of the transition to a boiling crisis time on the flow velocity during nucleate boiling of water on the heater's surface. The density dynamics of activating vaporization centers at different liquid flow velocities is shown. Mathematical modeling of the unsteady heating of a liquid flow was carried out, which showed the difference in the contribution made by convection into the heating of the near-wall layer of water and ethanol.

This research was performed at the Melentiev Energy Systems Institute SB RAS using the equipment of the HighTemperature Circuit Multi-Access Research Center and was funded by the Russian Foundation for Basic Research, Grant No. 18-29-10019.

\section{References}

1. J. Kim, Int. J. Multiph. Flow 35, 1067-1076 (2009)

2. D. A. Labuntsov, V. V. Yagov, Mehanika dvyhfaznyh sred (Izdatelstvo MEI, Moscow, 2000)

3. S. Glod, D. Poulikakos, Z. Zhao, G. Yadigarogly, Int. J. Heat Mass Transf. 45, 367-379 (2002)

4. V. I. Deev, K. V. Kutsenko, A. A. Lavruhin, K. O. Belyh, Proc.4th Russian National Conference on Heat Transfer 8, 81-84 (2010)

5. A. A. Levin, P. V. Khan, Int. J. Heat Mass Transf. 124, 876-883 (2018)

6. A. A. Levin, P. V. Khan, Appl. Therm. Eng., 149, pp. 1215-1222 (2019). 